\title{
Use of glucocorticoids in covid 19 patients: systematic review
}

\begin{abstract}
Summary
Introduction: The disease caused by sars-cov-2, covid 19 can generate, in its serious state, acute respiratory distress syndrome (ARDS), patients who must manage in-hospital, respiratory support, and hospitalization in an intermediate and intensive care unit (ICU), The effectiveness and reduction of morbidity and mortality in this group of patients has been demonstrated with the use of glucocorticoids of the dexamethasone type.
\end{abstract}

Objectives: To describe the action of glucocorticoids in reducing morbidity and mortality in severe covid 19 patients.

Methods: A search was carried out on the use of dexamethasone-type glucocorticoids in covid 19, in the PubMed, Medline and Google Scholar databases.

Results: Covid 19 with a severe course and ARDS can lead to a hyper-inflammatory state, in which the anti-inflammatory properties of steroids can be an effective therapeutic option. Steroid therapy has provento be useful in stabilizing patients hemodynamically, reducing prolonged stays in hospitalization services, intermediate care units, intensive care units, reducing the duration of mechanical ventilation, and reducing morbidity and mortality in this group of patients, serious patients.

Conclusion: Although upto now there is no established therapy that is $100 \%$ effective in the treatment of patients with severe covid, it is important to consider dexamethasone as a therapeutic strategy.

Keywords: dexamethasone, covid19, severe covid19, glucocorticoids, sars-cov-2
Volume 9 Issue I - 202 I

\section{Brigitte Nathalia Tascon Guevara}

Medical Specialist in Health Audit, Leader Science and Health, Colombia

Correspondence: Brigitte Nathalia Tascon Guevara, Medical Specialist in Health Audit, Leader Science and Health, Palmira, Valle del Cauca, Colombia, Tel +57 318376।643, Email bntgnat1987@hotmail.com

Received: December 222020 | Published: January 27, 2021

\section{Introduction}

The patho-physiological characteristics of severe Covid-19 are characterized by an acute pneumonic process with characteristics of extensive radiological opacity and, at autopsy, diffuse alveolar damage, inflammatory infiltrates, and microvascular thrombosis. Inflammatory organ injury can occur in severe Covid-19, with a subset of patients having elevated levels of inflammatory markers, including C-reactive protein, ferritin, interleukin-1, and interleukin-6. ${ }^{1}$ In the RECOVERY trial, dexamethasone was beneficial for participants treated for seven or more days in the symptomatic phase, with hypoxemia onset. ${ }^{2}$

A new phase of clinical trials is currently opening to evaluate the efficacy of drugs for COVID-19. In fact, a wide variety of drug screening assays are currently being conducted to explore the potential efficacy of new and old molecules in SARS-CoV-2. ${ }^{3-5}$ In a randomized clinical trial of 299 adults with moderate or severe ARDS due to COVID-19, dexamethasone associated with standard care compared to standard care alone significantly increased the number of days alive and without mechanical ventilation during the first 28 days. Dexamethasone was not associated with an increased risk of adverse events in this population of critically ill COVID-19 patients. ${ }^{6-8}$ Dexamethasone may be useful in the short term in severely intubated COVID-19 patients. $^{9,10}$

Proportional and absolute mortality rate reductions varied significantly by level of ventilation, dexamethasone reduced deaths by one third in patients receiving invasive mechanical ventilation, by one fifth in patients receiving oxygen without invasive mechanical ventilation, but it did not reduce mortality in patients who did not receive early ventilation. Before the announcement of the preliminary results of the RECOVERY trial, despite contraindications from the WHO and CDC, corticosteroids had been used globally in up to $50 \%$ of patients affected by COVID-19, particularly in China. All of these trials include severe and critical COVID-19 patients with lung involvement. Of the 5 studies ( 4 retrospective and 1 prospective) performed with cortico steroids, 3 studies have shown a benefit, while 2 studies showed no benefit, and there was a suggestion of significant harm especially in critical cases in one substudy. ${ }^{11,12}$

\section{Material and methods}

A search was carried out on the use of dexamethasone-type glucocorticoids in covid 19, in the PubMed, Medline and Google Scholar databases.

\section{Sensitivity analysis}

\section{Inclusion criteria:}

a. Patients diagnosed with covid 19

b. Patients older than 18 years with covid 19,

c. Use of glucocorticoids in the treatment of mild, moderate and or severe covid 19.

Exclusion criteria:

a. Diabetic patients with covid 19 ,

b. Children under 18 with a diagnosis of covid 19 .

From the clinical setting to the search strategy 


\begin{tabular}{|c|c|}
\hline Population & Patients diagnosed with covid 19 \\
\hline Intervention & $\begin{array}{l}\text { Use of dexamethasone or other } \\
\text { glucocorticoids in covid } 19\end{array}$ \\
\hline Comparator & Decrease in morbidity and mortality \\
\hline Outcome & Long stay decrease \\
\hline Search date range & March 2020- December 2020 \\
\hline Question type & Glucocorticoid treatment \\
\hline Type of study & Randomized clinical trials, meta-analysis. \\
\hline
\end{tabular}

\section{Research feasibility}

Evaluating the available bibliography, one finds extensive references, meta-analysis case reports, which show the effectiveness of the use of glucocortiocides in patients with moderate and severe covid19. With the execution of this topic review, no harm is generated to an individual, a community, or the environment, it seeks to evaluate the effectiveness of glucocorticoids in covid19. This research does not require financial support, a free access database isused to access information from open access sources PUBMED.

\section{Results}

Studies have shown that dexamethasone causes a significant improvement in the results of patients with severe COVID-19 mainly with ventilation.

\section{Conclusion}

It is important to describe that to date the world health organization (WHO) has not established a single treatment for the management of severe covid 19, or ARDS due to sars-cov-2, however according to the systematic review that is carried out, it is important to consider the use of glucocorticoids, especially dexamethasone, as an important therapeutic strategy that reduces morbidity and mortality, decreases prolonged stays, in seriously ill patients due to covid 19 .

\section{Acknowledgments}

None.

\section{Conflicts of interest}

Author declares that there is no conflict of interest.

\section{References}

1. The RECOVERY Collaborative Group. Dexamethasone in Hospitalized Patients with Covid-19-Preliminary Report; 2020.

2. Johnson RM. Dexamethasone in the management of covid-19. BMJ. 2020;370:m2648.

3. Ortolani C, Pastorello E. Hydroxychloroquine and dexamethasone in COVID-19: who won and who lost? Clinical and Molecular Allergy. 2020;18:17.

4. Pankaj Chibber, Syed Assim, Irfan Ahmed, et al. Advances in the possible treatment of COVID-19: A review. European Journal of Pharmacology. 2020;883:173372.

5. Solinas C, Perra L, Aiello M, et al. A critical evaluation of glucocorticoids in the management of severe COVID-19. Cytokine Growth Factor Rev. 2020;54:8-23.

6. Bruno M, Tomazini, Israel S Maia, et al, Effect of Dexamethasone on Days Alive and Ventilator-Free in Patients With Moderate or Severe Acute Respiratory Distress Syndrome and COVID-19. JAMA. 2020;324(13):1307-1316.

7. Mohammed Lester, Ali Sahin, Ali Pasyar. The use of dexamethasone in the treatment of COVID-19. Annals of Medicine and Surgery. 2020;56:218-219.

8. Kumar Singh A, Majumdar S, Singh R, e al. Role of corticosteroid in the management of COVID-19: A systemic review and a Clinician's perspective. Diabetes Metab Syndr. 2020;14(5):971-978.

9. Theoharides TC, Conti P. Dexamethasone for COVID-19? Not so fast. $J$ Biol Regul Homeost Agents. 2020;34(4):1241-1243.

10. Rayman G, Lumb AN, Kennon B, et al. Dexamethasone therapy in COVID-19 patients: implications and guidance for the management of blood glucose in people with and without diabetes. Diabet Med. 2021;38(1):e14378.

11. Chia Siang Kow, Syed Shahzad Hasan. Dexamethasone or hydrocortisone in COVID-19? Cleve Clin J Med. 2020;87(12):715.

12. Pfeifer M, Hamer O. COVID-19-Pneumonie. The internist. 2020;61:793-803. 